\title{
LEVITATION OF UNTETHERED STRESS-ENGINEERED MICROFLYERS USING THERMOPHORETIC (KNUDSEN) FORCE
}

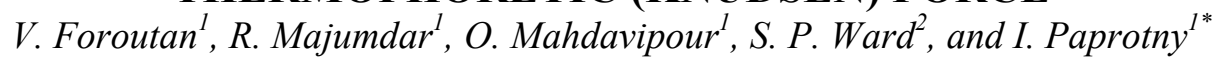 \\ ${ }^{1}$ Department of Electrical and Computer Engineering University of Illinois, Chicago, USA \\ ${ }^{2}$ Department of Electrical and Computer Engineering University of California, San Diego, USA
}

\section{ABSTRACT}

In this paper we present the theory and initial successful levitation experiments of untethered microscale structures (microflyers) using thermophoretic force. The microflyers consist of a $300 \mu \mathrm{m} \times 300 \mu \mathrm{m}$ sized chassis fabricated from polycrystalline silicon using a surface micromachining process. The devices are levitated using microfabricated heaters attached to an underlying substrate. A novel in-situ masked post-release stressengineering process is used to generate a concave upwards curvature of the flyers chassis, resulting in increased pitch and roll stability during flight, take-off, and landing.

\section{INTRODUCTION}

Several novel microscale mobile robotic systems have been developed in last decade, such as electrostatically driven stressengineered microrobots (MicroStressBots) [1], resonating stepping robots, stick-slip magnetic walkers and microscrew-based swimmers. Although flying microscale robots have not been previously demonstrated, levitation of untethered microstructures using the thermophoretic force has been previously proposed in [2].

In this paper we present the first experimental validation of successful untethered levitation of $300 \mu \mathrm{m} \times 300 \mu \mathrm{m}$ sized microscale structures (microflyers). The device is actuated (levitated) by Knudsen force generated by an underlying microfabricated heater. This is the first validation of potentially a new type of actuation mechanism for MEMS structures, which may pave way for new class of free-flying microscale robots.

The microflyer chassis was fabricated using a surface micromachining process. To stabilize the device during flight, the chassis was curved out-of-plane using an in-situ masked MEMS stress-engineering process.

\section{KNUDSEN FORCE}

When two separate objects at different temperatures are surrounded by a gas there is a force experienced by each of the objects known as the Knudsen force. The underlying mechanism for this force is the momentum imparted from the heated gas molecules onto the lower temperature object is greater than the momentum from the slower molecules striking the opposite side of the object. This force is strongest when the distance between intermolecular collisions and the separation distance are comparable i.e. their ratio is close to one. A relationship for the force between the temperature of the heated surface and a microcantilever was found by Passian [3] to be:

$$
F_{t h}=\frac{A_{c} P_{r}}{2}\left[\sqrt{\frac{T_{s}^{\prime}}{T_{r}}}+\sqrt{\frac{T_{c b}{ }^{\prime}}{T_{r}}}+\sqrt{\frac{T_{c t}{ }^{\prime}}{T_{r}}}-1\right]
$$

where $A_{c}$ is the surface area of the microflyer, $P_{r}$ is the pressure of the surrounding gas, $\operatorname{Tr}$ is the temperature of the surrounding gas, and $T_{s}{ }^{\prime}, T_{c b}{ }^{\prime}$, and $T_{c t}{ }^{\prime}$ are the temperatures of the reflected gas molecules after colliding with the substrate (heater), carrier bottom (bottom of the microflyer), and carrier top (top of the microflyer) respectively.

\section{DEVICE DESIGN}

The initially planar microrobot chassis was fabricated from polycrystalline silicon (polysilicon) using a multi-user MEMS fabrication process. The flyers and the heaters were released in hydrofluoric acid (49\% HF), and out-of-plane curvature was generated using a novel post-release stress engineering process. Figure 1 shows a scanning-electron micrograph of two of the stress-engineered microflyers that were successfully levitated during our experiments.

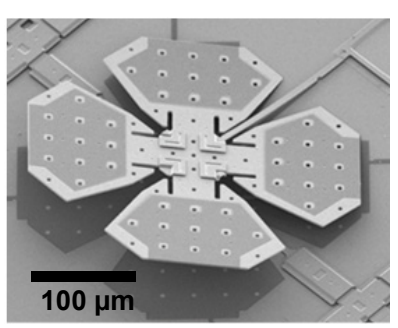

(a)

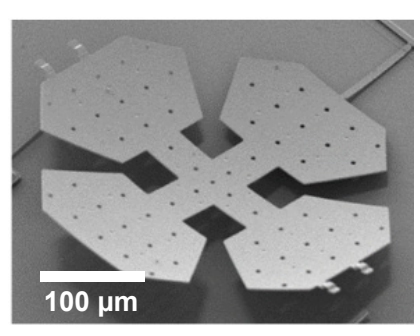

(b)
Figure 1: Scanning-electron micrograph of two types of stressengineered microflyers.

Figure 2 shows the curvature profile of five cantilevered test structures (SEM of the cantilevers is shown in the inset) exposed to $75 \mathrm{~nm}$ of thermally evaporated Chromium $(\mathrm{Cr})$ with an intrinsic compressive stress of $1.42 \times 10^{9} \mathrm{~Pa}$. The curvature can be controlled by varying the cross section (between cantilevers ii, iii, and iv), attaching fixed shadow masks (cantilever v), or using reconfigurable in-situ fabricated shadow masks (cantilever i). Sacrificial shadow-masks were also used to limit the curvature of the microflyer in Figure 1(a).

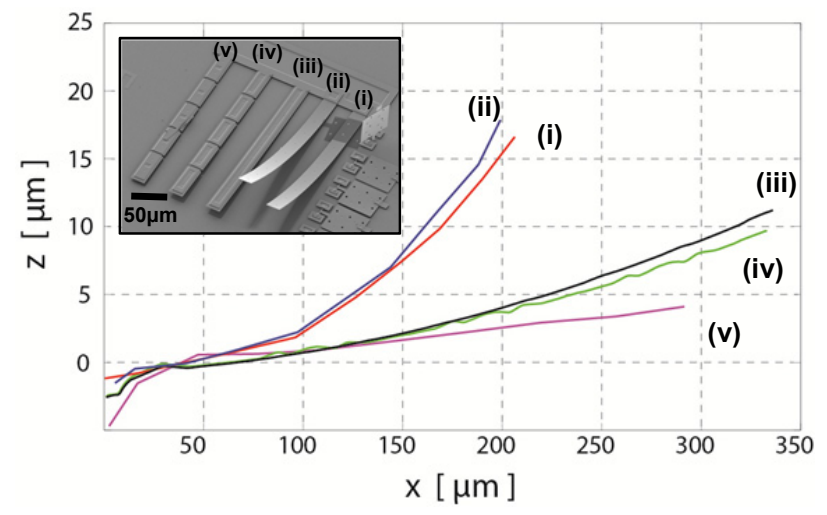

Figure 2: Height profiles for cantilevers (i-v) curved using in-situ masked stress engineering. Inset shows scanning-electron micrograph of the curved cantilevers.

The microflyers are fabricated attached to the transfer frames to enable successful transfer and placement on the heater prior to levitation. Figure 3 shows an optical micrograph of a transfer frame [1]. Microprobes inserted into the hinges of the transfer 
frame can be used to transport the microflyers between their location on the die and the fabricated heater.

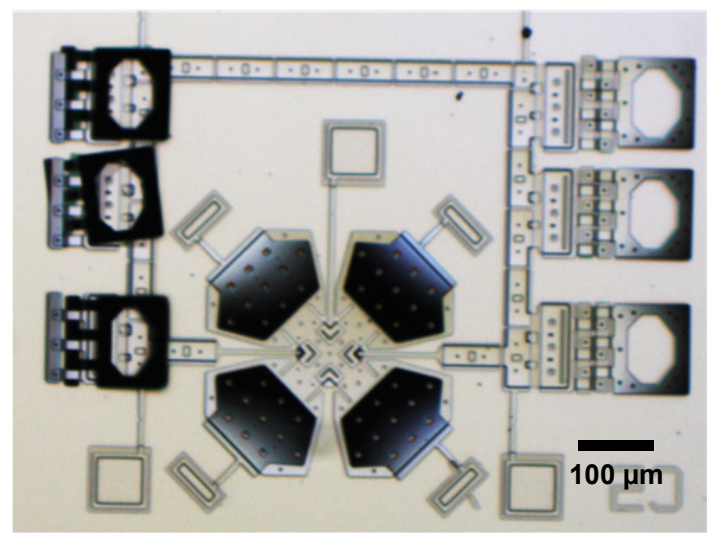

Figure 3: Optical micrograph of a stress-engineered microflyer attached to a transfer frame [1], which allows the flyer to be transported and deposited onto the heater. The frame chassis contains attached shadow masks, which protects it during the deposition of the stressor layer.

\section{EXPERIMENTAL RESULTS AND DISCUSSION}

We demonstrated both the actuation of microfabricated test structures using the Knudsen force, as well as the levitation of the untethered microrobot. Figure 4 shows the elevation of a tethered hinged test structure (as shown in the inset optical micrographs) using thermophoretic force generated by an underlying heater. These experimental results confirm our theoretical modeling of the thermophoretic force. Figure 5 shows images of microflyers prior to take-off, mid-flight, and after landing, in two separate levitation experiments. Because the heater was approximately the same size as the microflyer, the device would always translate towards its periphery. As expected, landing would occur as soon as significant part of the microflyer chassis would transit outside of the heated area.

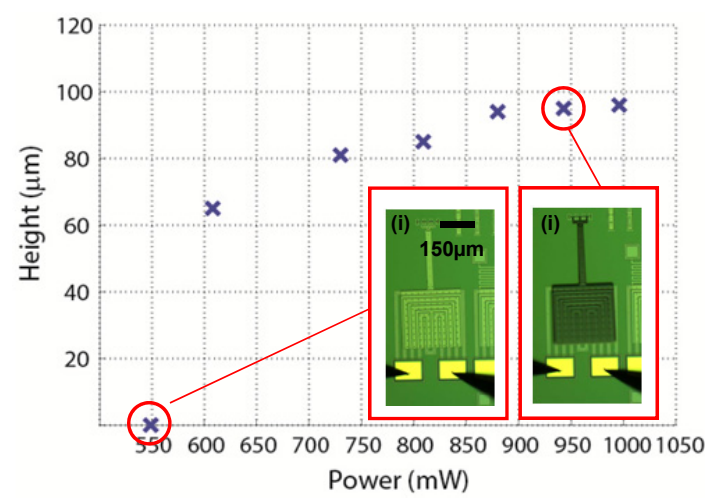

Figure 4: Tip height of a tethered hinged (i) test structure during the activation of an underlying heater. Inset shows the test structure before (left) and after (right) elevation close to its maximum height.

The results from repeated levitation experiments using both stressengineered and flat (not stress-engineered) microflyers are shown in Table 1. Successful microflyer levitation occurred in $85 \%$ of experiments using stress-engineered devices compared to $0 \%$ in the case of flat devices of otherwise similar geometry. In both cases, identical take-off power was applied to the heater. The experiments show that the stress-engineering process is instrumental in increasing the in-flight stability of the levitated microstructures.

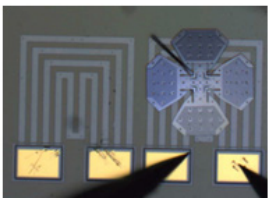

(a)

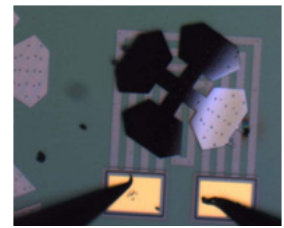

(a)

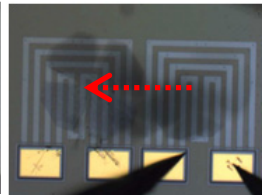

(b)

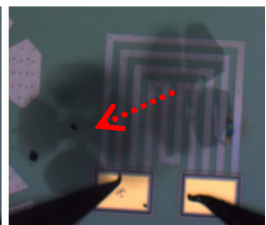

(b)

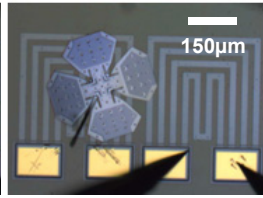

(c)

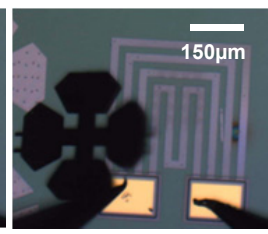

(c)
Figure 5: Two separate successful levitation experiments using two different types of microflyers (top and bottom). The optical micrographs show the flyers before (a), during (b) and after (c) levitation. Red arrow indicates the displacement of the flyers during the experiment.

Table 1: Results from levitation experiments using both stressengineered and flat microflyers.

\begin{tabular}{|l|c|c|}
\hline & $\begin{array}{c}\text { Stress- } \\
\text { Engineered }\end{array}$ & Flat \\
\hline Number of Trials & 7 & 5 \\
\hline Success Rate[\%] & $85 \%$ & $0 \%$ \\
\hline
\end{tabular}

\section{CONCLUSION}

We present the theory and experimental results demonstrating that concave stress-engineered structures are capable of achieving reliable untethered levitation using thermophoretic forces. Levitating microstructures present a novel mechanism for microrobot actuation, and enable many future applications in areas such as assembly, micromanipulation, and surveillance. As the thermophoretic force can be generated by heating the wings of the flyers rather than by using underlying heaters, the levitation mechanism demonstrated in this work may enable the development of future untethered aerial microscale robots.

\section{REFERENCES}

[1] B. R. Donald, C. G. Levey and I. Paprotny. "Planar Microassembly by Parallel Actuation of MEMS Microrobots." Journal of Microelectromechanical Systems (JMEMS) 17, 4 (2008), pp. 789-808.

[2] B. Gotsmann, D. Michel, and U. Duerig. "Controlled Thermophoresis as an Actuation Mechanism for Noncantilevered MEMS Devices." Journal of Microelectromechanical Systems (JMEMS) 17, 6 (2008), pp. 1287-1293.

[3] A. Passian, A. Wig, F. Meriaudeau, T.L. Ferrell and T. Thundat "Knudsen forces on microcantilevers." Journal of applied physics 92, 10 (2002), pp. 6326-6333.

\section{CONTACT}

*I. Paprotny, paprotny@uic.edu 